\title{
DIGITALCOMMONS
}

$5-1-2014$

\section{On the Gamma-Logistic Distribution}

Ayman Alzaatreh

Austin Peay State University, alzaatreha@apsu.edu

Indranil Ghosh

Austin Peay State University

Hassan Said

Austin Peay State University

Follow this and additional works at: http://digitalcommons.wayne.edu/jmasm

\section{Recommended Citation}

Alzaatreh, Ayman; Ghosh, Indranil; and Said, Hassan (2014) "On the Gamma-Logistic Distribution," Journal of Modern Applied Statistical Methods: Vol. 13 : Iss. 1 , Article 5.

DOI: $10.22237 /$ jmasm/1398917040

Available at: http://digitalcommons.wayne.edu/jmasm/vol13/iss1/5 


\section{On the Gamma-Logistic Distribution}

Ayman Alzaatreh

Austin Peay State University

Clarksville, TN
Indranil Ghosh

Austin Peay State University

Clarksville, TN
Hassan Said

Austin Peay State University

Clarksville, TN

A new generalization of the logistic distribution is defined and studied, namely, the gamma-logistic distribution. Various properties of the gamma-logistic are obtained. The structural analysis of the distribution includes moments, mode, quantiles, skewness, kurtosis, Shannon's entropy and order statistics. The method of maximum likelihood estimation is proposed for estimating the model parameters. For illustrative purposes, a real data set is analyzed as an application of the gamma-logistic distribution.

Keywords: $\quad$ gamma-logistic distribution, $T$ - $X$ family of distributions, Shannon's entropy, reliability parameter, order statistics

\section{Introduction}

The armory of statistical distributions is truly illimitable. New distributions are being unearthed literally on a weekly basis elicited by either theoretical considerations or by pressing practical applications or both. A new class of mixtures of two absolutely continuous distributions is investigated in this article, with the primary objective of exploring its enhanced flexibility in modeling skewed data.

The simplicity of the logistic distribution and its importance as a growth curve has attracted many researchers to study this distribution. Also, the limitation of the shape of the logistic distribution merits further investigation to various other different types of generalized logistic distribution. Many other generalized distributions obtained from the logistic distribution were introduced in the literature to study its skewness properties and to examine its flexibility in modeling skewed data. It is noteworthy to mention that other generalizations of logistic distribution exists in the literature such as Types I, II, III and IV generalized logistic distributions (Johnson at el., 1994). Proposed here is a new

Drs. Alzaatreh and Ghosh are both Assistant Professors in the Department of Mathematics and Statistics. Email Dr. Alzaatreh at: alzaatreha@apsu.edu.Dr.Hassaan is an Associate Professor in the College of Business. 


\section{ON THE GAMMA-LOGISTIC DISTRIBUTION}

distribution, mixing two absolutely continuous distributions, Gamma and logistic, following the idea discussed in Alzaatreh, et al. (2013b).

Let $F(x)$ be the cumulative distribution function (CDF) of any random variable $X$ and $r(t)$ be the probability density function (PDF) of a random variable $T$ defined on $[0, \infty)$. The CDF of the $T-X$ family of distributions defined by Alzaatreh, et al. (2013b) is given by

$$
G(x)=\int_{0}^{-\log (1-F(x))} r(t) d t
$$

When $X$ is a continuous random variable, the probability density function of the $T$ - $X$ family is

$$
g(x)=\frac{f(x)}{1-F(x)} r(-\log (1-F(x)))
$$

Recently, many generalized distributions have been generated from the $T-X$ family of distributions such as Weibull-Pareto distribution (Alzaatreh et al., 2013a), gamma-Pareto distribution (Alzaatreh et al., 2012a), gamma-Pareto IV (Alzaatreh and Ghosh, 2013), gamma-half normal distribution (Alzaatreh and Knight, 2013) and the exponentiated-exponential geometric distributions (Alzaatreh et al., 2012b). For more information about methods for generating univariate continuous distributions, one may refer to Lee et al. (2013).

If a random variable $T$ follows the gamma distribution with parameters $\alpha$ and $\beta, r(t)=\left(\beta^{\alpha} \Gamma(\alpha)\right)^{-1} t^{\alpha-1} e^{-t / \beta}, t \geq 0$, the definition in (2) leads to the gamma- $X$ family with the PDF

$$
g(x)=\frac{1}{\Gamma(\alpha) \beta^{\alpha}} f(x)(-\log (1-F(x)))^{\alpha-1}(1-F(x))^{\frac{1}{\beta}-1} .
$$

If $X$ follows a logistic distribution with parameters $\theta, F(x)=1-\left(1+e^{x / \theta}\right)^{-1}, x \in \mathbb{R}$, then (3) reduces to

$$
g(x)=\frac{1}{\theta \beta^{\alpha} \Gamma(\alpha)} e^{x / \theta}\left(\log \left(1+e^{x / \theta}\right)\right)^{\alpha-1}\left(1+e^{x / \theta}\right)^{-1-1 / \beta}, x \in \mathbb{R} ; \alpha, \beta, \theta>0 .
$$




\section{ALZAATREH ET AL}

Note that when $\alpha=\beta=1$, the PDF in (4) reduces to the logistic distribution. When $\beta=1$ and $\alpha=n \in \mathrm{N}$, the PDF in (4) reduces to the density function of the upper record values, $X_{U(n)}$, arising from a sequence $\left\{X_{i}\right\}_{i=1}^{n}$ of identically independent logisitc random variables (Johnson, et al., 1994, pp. 135). From (4), the CDF of the gamma-logistic distribution can be written as

$$
G(x)=\frac{1}{\Gamma(\alpha)} \gamma\left(\alpha, \beta^{-1} \log \left(1+e^{x / \theta}\right)\right)
$$

where $\gamma(a, x)=\int_{0}^{x} t^{a-1} e^{-t} d t$ is the lower incomplete gamma function.

\section{Some properties of the gamma-logistic distribution}

The following lemma provides a characterization of the gamma-logistic distribution which establishes the relation between gamma-logistic and gamma distributions.

\section{Lemma 1 (Transformation)}

If a random variable $X$ follows the gamma distribution with parameters $\alpha$ and $\beta$, then $Y=\theta \log \left(e^{X}-1\right)$ follows gamma-logistic distribution with parameters $\alpha, \beta$ and $\theta$.

Proof: The result follows by using the transformation technique.

\section{Lemma 2 (Mode)}

The mode of the gamma-logistic distribution is the solution of the equation $k(x)=0$, where $k(x)=\log \left(1+e^{x / \theta}\right)\left(1-\beta^{-1} e^{x / \theta}\right)+(\alpha-1) e^{x / \theta}$.

Proof: Setting $g^{\prime}(x)=0$ is equivalent to

$\theta^{-2} \beta^{-\alpha}(\Gamma(\alpha))^{-1} e^{x / \theta}\left(\log \left(1+e^{x / \theta}\right)\right)^{\alpha-2}\left(1+e^{x / \theta}\right)^{-2-1 / \beta} k(x)=0$, where $k(x)=\log \left(1+e^{x / \theta}\right)\left(1-\beta^{-1} e^{x / \theta}\right)+(\alpha-1) e^{x / \theta}$. Hence, the critical values of $g(x)$ is the solution of $k(x)=0$. 


\section{ON THE GAMMA-LOGISTIC DISTRIBUTION}

In Figures 1 and 2, various graphs of $g(x)$ are provided for different parameter values. The plots indicate that the gamma-logistic distribution is unimodal and can be symmetric, right-skewed or left-skewed.

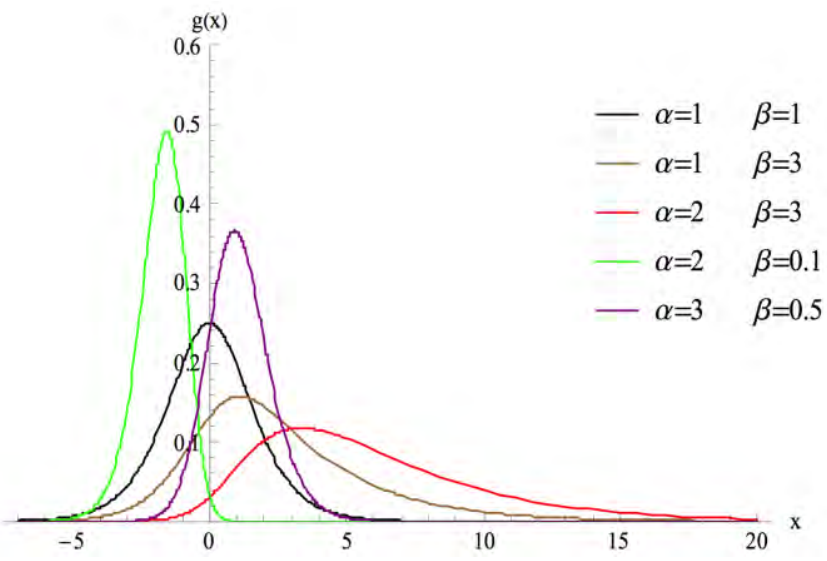

Figure 1. Graphs of the gamma-logistic PDF for various choices of $\alpha$ and $\beta$ when $\theta=1$.

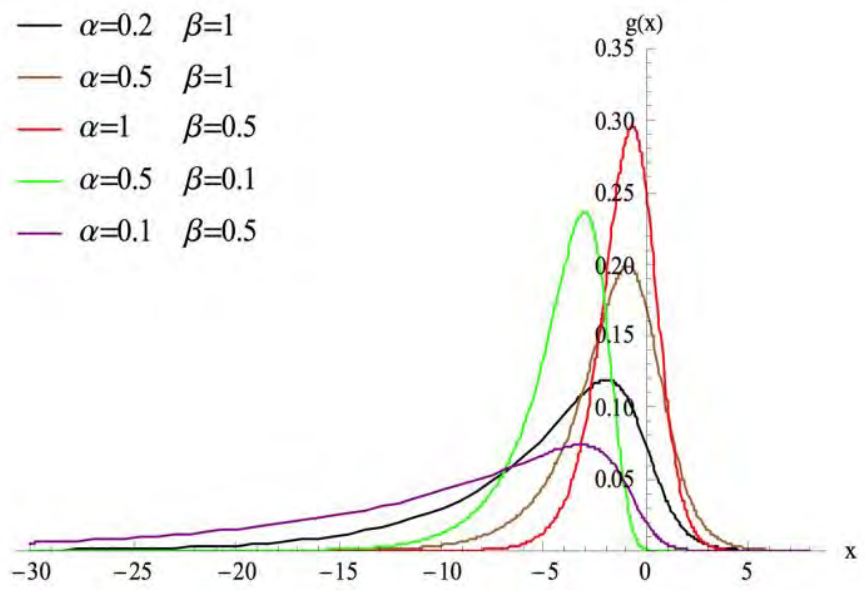

Figure 2. Graphs of the gamma-logistic PDF for various choices of $\alpha$ and $\beta$ when $\theta=1$. 


\section{ALZAATREH ET AL}

\section{Lemma 3}

Let $Q(\lambda), 0<\lambda<1$, denotes the quantile function for the gamma-logistic distribution. Then $Q(\lambda)$ can be written as

$$
Q(\lambda)=\theta \log \left(e^{\beta \gamma^{-1}(\alpha, \lambda \Gamma(\alpha))}-1\right)
$$

Proof: $\quad$ The result follows by using $G(Q(\lambda))=\lambda$ in (5) and then solving it for $Q(\lambda)$

The Shannon's entropy (Shannon, 1948) plays an important role in information theory and it is used as a measure of uncertainty. Shannon's entropy for a random variable $X$ with PDF $g(x)$ is defined as $E[-\log (g(X))]$. According to Alzaatreh, et al. (2013b), the Shannon's entropy for the gamma- $X$ family can be written as

$$
-E\left[\log \left(f\left(F^{-1}\left(1-e^{-T}\right)\right)\right)\right]+\alpha(1-\beta)+\log \beta+\log (\Gamma(\alpha))+(1-\alpha) \psi(\alpha),
$$

where $F($.$) and f($.$) are the \mathrm{CDF}$ and $\mathrm{PDF}$ of the Transformer family respectively, and $T$ follows gamma distibution with parameters $\alpha$ and $\beta$, and $\psi($.$) is the digamma function.$

The following theorem defines expression for the Shannon's entropy for the gamma-logistic distribution.

\section{Theorem 1}

The Shannon's entropy for the random variable $X$ which follows a gamma-logistic distribution is given by

$$
\eta_{x}=\alpha+\log (\beta \theta)+\log \Gamma(\alpha)+(1-\alpha) \psi(\alpha)+\sum_{k=1}^{\infty} \frac{1}{k(1+\beta k)^{\alpha}}
$$

Proof: In this case, $F(X)=1-\left(1+e^{X / \theta}\right)^{-1}$. So that

$$
\log \left\{f\left(F^{-1}\left(1-e^{T}\right)\right)\right\}=\log \left(e^{T}-1\right)-2 T-\log \theta=\log \left(1-e^{-T}\right)-T-\log \theta
$$




\section{ON THE GAMMA-LOGISTIC DISTRIBUTION}

where $T$ follows the gamma distribution with parameters $\alpha$ and $\beta$. Now,

consider $E\left(\log \left(1-e^{-T}\right)\right)$. Using the Taylor's series expansion of $\log \left(1-e^{-T}\right)$, one can get

$$
\begin{aligned}
E\left(\log \left(1-e^{-T}\right)\right) & =-\sum_{k=1}^{\infty} k^{-1} E\left(e^{-k T}\right)=-\frac{1}{\beta^{\alpha} \Gamma(\alpha)} \sum_{k=1}^{\infty} k^{-1} \int_{0}^{\infty} e^{-k t} e^{t / \beta} t^{\alpha-1} d t \\
& =-\sum_{k=1}^{\infty} \frac{1}{k(1+\beta k)^{\alpha}}
\end{aligned}
$$

$$
\text { Hence, }-E\left[\log \left(f\left(F^{-1}\left(1-e^{-T}\right)\right)\right)\right]=\sum_{k=1}^{\infty} \frac{1}{k(1+\beta k)^{\alpha}}+E(T)+\log \theta \text {. }
$$

The result in (8) follows by using the fact that $E(T)=\alpha \beta$ and substituting (10) in (7).

\section{Moments and mean deviations}

The moment generating function for the gamma-logistic distribution is given by

$$
M_{X}(t)=E\left(e^{t X}\right)=\frac{1}{\beta^{\alpha} \theta \Gamma(\alpha)} \int_{-\infty}^{\infty} e^{x(t+1 / \theta)}\left(\log \left(1+e^{x / \theta}\right)\right)^{\alpha-1}\left(1+e^{x / \theta}\right)^{-1-1 / \beta} d x
$$

On using the substitution $u=\log \left(1+e^{x / \theta}\right)$, (11) can be written as

$$
M_{X}(t)=\frac{1}{\beta^{\alpha} \Gamma(\alpha)} \int_{0}^{\infty}\left(e^{u}-1\right)^{t \theta} u^{\alpha-1} e^{-u / \beta} d u
$$

On writing $\left(e^{u}-1\right)^{t \theta}=e^{t u \theta}\left(1-e^{-u}\right)^{t \theta}$ and using the generalized binomial expression of $\left(1-e^{-u}\right)^{t \theta}=\sum_{k=0}^{\infty} \frac{(-1)^{k}(t \theta)_{(k)} e^{-k u}}{k !}$, one can get

$$
M_{X}(t)=\sum_{k=0}^{\infty} \frac{(-1)^{k}(t \theta)_{(k)}}{k !(\beta(k-t \theta)+1)^{\alpha}}
$$

provided that $t<\theta^{-1}$, and $(t \theta)_{(k)}=t \theta(t \theta-1) \cdots(t \theta-k+1)$. 


\section{ALZAATREH ET AL}

A series expression for the $r^{\text {th }}$ moments of the gamma-logistic distribution can be obtained by using the fact that $E\left(X^{r}\right)=\left.\frac{d^{r}}{d t^{r}} M_{X}(t)\right|_{t=0}$.

Skewness and kurtosis of a distribution can be measured by $\beta_{1}=\mu_{3} / \sigma^{3}$ and $\beta_{2}=\mu_{4} / \sigma^{4}$, respectively. When the distribution is symmetric, $\beta_{1}=0$ and when the distribution is right (or left) skew, $\beta_{1}>0$ (or $\beta_{1}<0$ ). As $\beta_{2}$ increases the tail of the distribution becomes heavier. To investigate the effect of the two shape parameters $\alpha$ and $\beta$ on the gamma-logistic distribution, $\beta_{1}$ and $\beta_{2}$ were computed for different values of $\alpha$ and $\beta$. Figures 3 and 4 display the skewness and the kurtosis for the gamma-logistic distribution when $\theta=1$. From Figure 3, the gamma-logistic distribution can be left skewed, right skewed or symmetric. Also, for fixed value of $\alpha$, the skewness is an increasing function of $\beta$. As shown in Figure 4 , as $\alpha$ gets smaller, the kurtosis of the gamma-logistic distribution increases rapidly as $\beta$ increases.

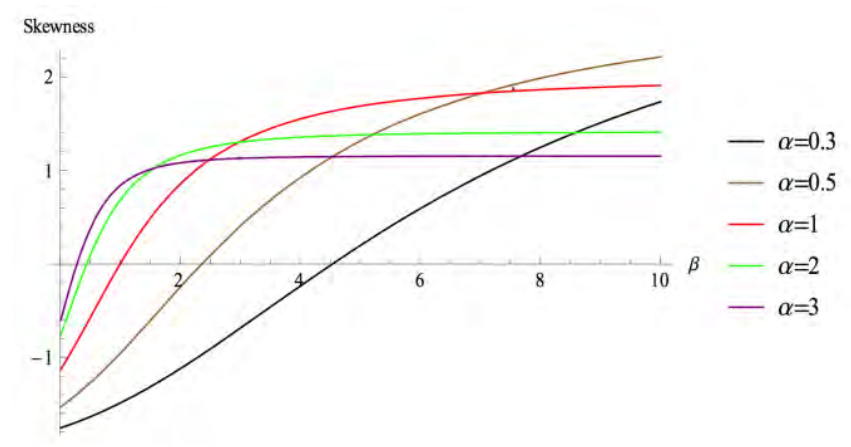

Figure 3. Skewness graph for gamma-logistic distribution when $\theta=1$.

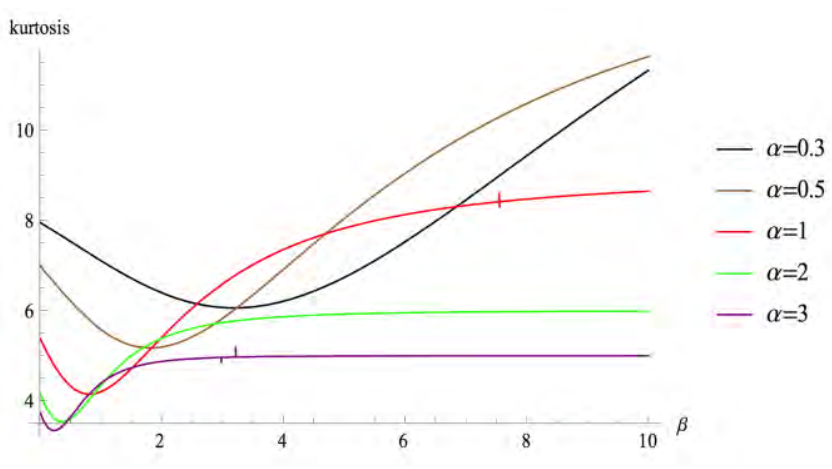

Figure 4. Kurtosis graph for gamma-logistic distribution when $\theta=1$. 


\section{ON THE GAMMA-LOGISTIC DISTRIBUTION}

The deviation from the mean and the deviation from the median are used to measure the dispersion and the spread in a population. If we denote the median by $M$, then the mean deviation from the mean, $D(\mu)$, and the mean deviation from the median, $D(M)$, can be written as

$$
\begin{aligned}
& D(\mu)=E|X-\mu|=2 \mu G(\mu)-2 \int_{-\infty}^{\mu} x g(x) d x . \\
& D(M)=E|X-M|=\mu-2 \int_{-\infty}^{M} x g(x) d x .
\end{aligned}
$$

Now, consider

$$
\begin{aligned}
I_{m} & =\int_{-\infty}^{m} x g(x) d x \\
& =\frac{1}{\theta \beta^{\alpha} \Gamma(\alpha)} \int_{-\infty}^{\infty} x e^{x / \theta}\left(\log \left(1+e^{x / \theta}\right)\right)^{\alpha-1}\left(1+e^{x / \theta}\right)^{-1-1 / \beta} d x
\end{aligned}
$$

Using the substitution $u=\log \left(1+e^{x / \theta}\right)$ in (15) results in

$$
I_{m}=\frac{\theta}{\beta^{\alpha} \Gamma(\alpha)}\left(\int_{0}^{\log \left(1+e^{m / \theta}\right)} u^{\alpha} e^{-u / \beta} d u+\int_{0}^{\log \left(1+e^{m / \theta}\right)} \log \left(1-e^{-u}\right) u^{\alpha-1} e^{-u / \beta} d u\right)
$$

Using the Taylor series expansion of $\log \left(1-e^{-u}\right)$ results in

$$
I_{m}=\frac{\theta}{\Gamma(\alpha)}\left\{\gamma\left(\alpha+1, \beta^{-1} \log \left(1+e^{m / \theta}\right)\right)-\sum_{k=1}^{\infty} \frac{\beta^{k+1}}{k} \gamma\left(\alpha+k, \beta^{-1} \log \left(1+e^{m / \theta}\right)\right)\right\}
$$

Using equations (5) and (16), the mean deviation from the mean and the mean deviation from the median are

$$
D(\mu)=2 \mu \frac{\gamma\left(\alpha, \beta^{-1} \log \left(1+e^{\mu / \theta}\right)\right)}{\Gamma(\alpha)}-2 I_{\mu} \text { and } D(M)=\mu-2 I_{M}
$$




\section{ALZAATREH ET AL}

\section{Mean residual life function and Reliability parameter}

Let $X$ be a random variable with cumulative distribution function CDF $F$ such that $E(X)<\infty$. The mean residual life (MRL) function $\xi(x)$ of $X$ is defined by $\xi(x)=E(X-x \mid X>x)$. The MRL function, also known as expected remaining life function or mean excess function, has been extensively studied in lifetime variables context. It plays a major role in many fields such as industrial reliability, life insurance and biomedical science. For more information about the MRL function, see Kotz and Shanbhag (1980), Hall and Wellner (1979) and Guess and Proschan (1985). The next theorem demonstrates the expression of the MRL function for the Gamma-logistic distribution.

\section{Theorem 2}

Let $X$ be a random variable which follows the gamma-logistic distribution with parameters $\alpha, \beta$ and $\theta$. Then the MRL function is given by

$$
\xi(x)=\frac{\theta}{\Gamma(\alpha)}\left\{\beta \Gamma\left(\alpha+1, \beta^{-1} \log \left(1+e^{x / \theta}\right)\right)-\sum_{k=1}^{\infty} \frac{\Gamma\left(\alpha,\left(\beta^{-1}+k\right) \log \left(1+e^{x / \theta}\right)\right)}{k(\beta k+1)^{\alpha}}\right\}-x,
$$

where $\Gamma(x, a)=\int_{x}^{\infty} t^{a-1} e^{-t} d t$ is the upper incomplete gamma function.

\section{Proof: $\quad$ From (4),}

$$
E(X \mid X>x)=\frac{1}{\theta \beta^{\alpha} \Gamma(\alpha)} \int_{x}^{\infty} t e^{t / \theta}\left(\log \left(1+e^{t / \theta}\right)\right)^{\alpha-1}\left(1+e^{t / \theta}\right)^{-1-1 / \beta} d t
$$

On using the substitution $u=\log \left(1+e^{t / \theta}\right)$, (18) reduces to

$$
\begin{aligned}
E(X \mid X>x) & =\frac{\theta}{\beta^{\alpha} \Gamma(\alpha)} \int_{\log \left(1+e^{x / \theta}\right)}^{\infty} u^{\alpha-1} e^{-u / \beta} \log \left(e^{u}-1\right) d u \\
& =\frac{\theta}{\beta^{\alpha} \Gamma(\alpha)} \int_{\log \left(1+e^{x / \theta}\right)}^{\infty} u^{\alpha-1} e^{-u / \beta}\left(u-\sum_{k=1}^{\infty} e^{-k u} / k\right) d u
\end{aligned}
$$

The result follows from equation (19). 


\section{ON THE GAMMA-LOGISTIC DISTRIBUTION}

The reliability parameter $R$ is defined as $R=P(X>Y)$, where $X$ and $Y$ are independent random variables. Many applications of the reliability parameter have appeared in the literature such as the area of classical stress-strength model and the breakdown of a system having two components. More applications of the reliability parameter can be found in Hall (1984) and Weerahandi and Johnson (1992). If $X$ and $Y$ are two continuous independent random variables with CDFs $F_{1}(x)$ and $F_{2}(y)$ and their PDFs $f_{1}(x)$ and $f_{2}(y)$ respectively. Then the

reliability parameter $R$ can be written as $R=P(X>Y)=\int_{-\infty}^{\infty} F_{2}(t) f_{1}(t) d t$.

The following theorem provides an expression for the reliability parameter $R$ where the parameter $\theta$ is fixed.

\section{Theorem 3}

Suppose that $X$ and $Y$ are two independent gamma-logistic random variables with parameters $\left(\alpha_{1}, \beta_{1}, \theta\right)$ and $\left(\alpha_{2}, \beta_{2}, \theta\right)$ respectively. Then

$$
P(X>Y)=\frac{1}{\Gamma\left(\alpha_{1}\right) \Gamma\left(\alpha_{2}\right)} \sum_{k=0}^{\infty}\left(\frac{\beta_{1}}{\beta_{2}}\right)^{\alpha_{2}+k} \frac{(-1)^{k} \Gamma\left(\alpha_{1}+\alpha_{2}+k\right)}{k !\left(k+\alpha_{2}\right)} .
$$

Proof: $\quad$ From (4) and (5),

$$
P(X>Y)=\frac{1}{\theta \beta_{1}^{\alpha_{1}} \Gamma\left(\alpha_{1}\right) \Gamma\left(\alpha_{2}\right)} \int_{-\infty}^{\infty} \gamma\left(\alpha_{2}, \beta_{2}^{-1} \log \left(1+e^{t / \theta}\right)\right) e^{t / \theta}\left(1+e^{t / \theta}\right)^{-1-1 / \beta_{1}}\left(\log \left(1+e^{t / \theta}\right)\right)^{\alpha_{1}-1} d t .
$$

On using the following series expansion from Nadarajah and Pal (2008),

$$
\gamma(\alpha, x)=\sum_{k=0}^{\infty}(-1)^{k} \frac{x^{k+\alpha}}{k !(k+\alpha)}
$$

and then the substitution $w=\log \left(1+e^{t / \theta}\right)$, equation (21) can be written as

$$
P(X>Y)=\frac{1}{\beta_{1}^{\alpha_{1}} \Gamma\left(\alpha_{1}\right) \Gamma\left(\alpha_{2}\right)} \sum_{k=0}^{\infty} \frac{(-1)^{k} \beta_{2}^{-\alpha_{2}-k}}{k !\left(k+\alpha_{2}\right)} \int_{0}^{\infty} w^{\alpha_{1}+\alpha_{2}+k-1} e^{-w / \beta_{1}} d w
$$

Equation (23) reduces to (20). 


\section{ALZAATREH ET AL}

\section{Order Statistics for the gamma-logistic distribution}

Consider the general $r^{\text {th }}$ order statistic and the asymptotic distributions of the sample minimum and maximum when a random sample of size $n$ is drawn from the gamma-logistic. From (5), the density function of the $r^{\text {th }}$ order statistic, $X_{r: n}$, is given by

$$
\begin{aligned}
f_{X_{r n}}(x) & =\frac{1}{B(r, n-r+1)}(G(x))^{r-1}(1-G(x))^{n-r} g(x) \\
& =\frac{1}{B(r, n-r+1)} g(x) \sum_{j=0}^{n-r}(-1)^{j}\left(\begin{array}{c}
n-r \\
j
\end{array}\right)\left\{\frac{\gamma\left(\alpha, \beta^{-1} \log \left(1+e^{x / \theta}\right)\right)}{\Gamma(\alpha)}\right\}^{r+j-1}, x \in \mathbb{R} .
\end{aligned}
$$

On using (22), the PDF of $X_{r: n}$ can be written as

$$
\begin{aligned}
f_{r: n}(x) & =\frac{1}{B(r, n-r+1)} g(x) \sum_{j=0}^{n-r}(-1)^{j}\left(\begin{array}{c}
n-r \\
j
\end{array}\right)\left\{\sum_{k=0}^{\infty} \frac{(-1)^{k}}{\Gamma(\alpha) \beta^{k+\alpha}} \frac{\left(\log \left(1+e^{x / \theta}\right)\right)^{k+\alpha}}{k !(k+\alpha)}\right\}^{r+j-1} \\
& =\frac{1}{B(r, n-r+1)} g(x) \sum_{j=0}^{n-r} \sum_{k_{1}=0}^{\infty} \cdots \sum_{k_{r+j-1}=0}^{\infty}(-1)^{j+s_{k}}\left(\begin{array}{c}
n-r \\
j
\end{array}\right) \frac{\left(\log \left(1+e^{x / \theta}\right)\right)^{s_{k}+(r+j-1) \alpha}}{(\Gamma(\alpha))^{r+j-1} \beta^{s_{k}+(r+j-1) \alpha} p_{k}} \\
& =\frac{1}{B(r, n-r+1)} \sum_{j=0}^{n-r} \sum_{k_{1}=0}^{\infty} \cdots \sum_{k_{r+j-1}=0}^{\infty}(-1)^{j+s_{k}}\left(\begin{array}{c}
n-r \\
j
\end{array}\right) \frac{\Gamma\left(s_{k}+(r+j) \alpha\right)}{(\Gamma(\alpha))^{r+j} p_{k}} g\left(x \mid s_{k}+(r+j) \alpha, \beta, \theta\right),
\end{aligned}
$$

where $s_{k}=\sum_{i=1}^{r+j-1} k_{i}$ and $p_{k}=\prod_{i=1}^{r+j-1} k_{i} !\left(k_{i}+\alpha\right)$.

From (24), note that the PDF of the $r^{\text {th }}$ order statistic $X_{r: n}$ can be expressed as infinite sums of the gamma-logistic PDFs.

To study the asymptotic distributions of the sample minimum $X_{1: n}$ and the sample maximum $X_{n: n}$, use Theorem 8.3.6 of Arnold et al. (2008) as follows: Since $G^{-1}(0)=-\infty$, it follows from the Theorem that the asymptotic distribution of the sample minimum $X_{1: n}$ will be of the Weibull type with parameter $\delta>0$ if $\lim _{\varepsilon \rightarrow 0_{+}} \frac{G(\varepsilon x)}{G(\varepsilon)}=x^{\delta}$, for all $x>0$. By using the L'Hospital's rule, it can be easily 


\section{ON THE GAMMA-LOGISTIC DISTRIBUTION}

shown that $\lim _{\varepsilon \rightarrow 0_{+}} \frac{G(\varepsilon x)}{G(\varepsilon)}=x \lim _{\varepsilon \rightarrow 0_{+}} \frac{g(\varepsilon x)}{g(\varepsilon)}=x^{\alpha}$. Hence, the asymptotic distribution of $X_{1: n}$ is of the Weibull type with shape parameter $\alpha$. The asymptotic distribution of the sample maximum $X_{n: n}$ can be viewed as $G_{n}(x)$ where $G_{n}(x)=1-G_{1}(-x)$, where $G_{1}(x)$ is the CDF of $X_{1: n}$.

\section{Maximum likelihood estimation}

Let a random sample of size $n$ be taken from the gamma-logistic distribution. The log-likelihood function for the gamma-logistic distribution in (4) is given by

$$
\begin{array}{rr}
\ell=-n \log \theta-n \log \Gamma(\alpha)-n \alpha \log \beta+\frac{n \bar{X}}{\theta} & -(1+1 / \beta) \sum_{i=1}^{n} \log \left(1+e^{X_{i} / \theta}\right) \\
& +(\alpha-1) \sum_{i=1}^{n} \log \left(\log \left(1+e^{X_{i} / \theta}\right)\right.
\end{array}
$$

The derivatives of (25) with respect to $\alpha, \beta$ and $\theta$ are given by

$$
\begin{aligned}
& \frac{\partial \ell}{\partial \alpha}=-n \psi(\alpha)-n \log \beta+\sum_{i=1}^{n} \log \left(\log \left(1+e^{X_{i} / \theta}\right)\right) . \\
& \frac{\partial \ell}{\partial \beta}=-\frac{n \alpha}{\beta}+\frac{1}{\beta^{2}} \sum_{i=1}^{n} \log \left(1+e^{X_{i} / \theta}\right) . \\
& \frac{\partial \ell}{\partial \theta}=-\frac{n}{\theta}-\frac{n \bar{X}}{\theta^{2}}-\frac{1}{\theta^{2}} \sum_{i=1}^{n} \frac{X_{i} e^{X_{i} / \theta}}{\left(1+e^{X_{i} / \theta}\right)}\left(\frac{\alpha-1}{\log \left(1+e^{X_{i} / \theta}\right)}+\frac{1}{\beta}+1\right) .
\end{aligned}
$$

The MLE of $\hat{\alpha}, \hat{\beta}$ and $\hat{\theta}$ are obtained by setting (26), (27) and (28) to zero and solving them iteratively.

\section{Application}

The gamma-logistic is applied to a data set from Brinbaum and Saunders (1969). The data set represents the fatigue life of 6061-T6 aluminum coupons cut parallel 


\section{ALZAATREH ET AL}

with the direction of rolling and oscillated at 18 cycles per second. The data set was fitted in Table 1 and the result compared with the logistic, Type I logistic, Type II logistic, Type III logistic and Type IV logistic distributions (Johnson et al., 1994). The maximum likelihood estimates, the log-likelihood value, the AIC (Akaike Information Criterion), the Kolmogorov-Smirnov test statistic (K-S) and the p-value for the K-S statistics for the fitted distributions are reported in Table 2. The results from Table 2 indicate that the gamma-logistic distribution provides the best fit among the distributions. Also, the K-S p-values indicate that only gamma-logistic and Type I logistic distributions provide an adequate fit to the data. The empirical and the fitted cumulative distribution functions are displayed in Figure 5. This figure supports the results in Table 2.

Table 1. Fatigue Life of 6061-T6 Aluminum

\begin{tabular}{rrrrrrrrrr}
\hline 70 & 90 & 96 & 97 & 99 & 100 & 103 & 104 & 104 & 105 \\
107 & 108 & 108 & 108 & 109 & 109 & 112 & 112 & 113 & 114 \\
114 & 114 & 116 & 119 & 120 & 120 & 120 & 121 & 121 & 123 \\
124 & 124 & 124 & 124 & 124 & 128 & 128 & 129 & 129 & 130 \\
130 & 130 & 131 & 131 & 131 & 131 & 131 & 132 & 132 & 132 \\
133 & 134 & 134 & 134 & 134 & 134 & 136 & 136 & 137 & 138 \\
138 & 138 & 139 & 139 & 141 & 141 & 142 & 142 & 142 & 142 \\
142 & 142 & 144 & 144 & 145 & 146 & 148 & 148 & 149 & 151 \\
151 & 152 & 155 & 156 & 157 & 157 & 157 & 157 & 158 & 159 \\
162 & 163 & 163 & 164 & 166 & 166 & 168 & 170 & 174 & 196 \\
212 & & & & & & & & & \\
\hline
\end{tabular}

Table 2. Parameter estimates for the fatigue life of 6061-T6 aluminum coupons data

\begin{tabular}{|c|c|c|c|c|c|c|c|}
\hline Distribution & $\hat{\alpha}$ & $\hat{\beta}$ & $\hat{\theta}$ & $\begin{array}{r}\text { Log } \\
\text { likelihood }\end{array}$ & AIC & KS & $\begin{array}{r}\text { K-S } \\
\text { p-value }\end{array}$ \\
\hline Logistic & - & - & 87.1321 & -646.5934 & 1295.1868 & 0.7209 & 0.0000 \\
\hline Logistic I & 288.2700 & - & 21.7088 & -462.3355 & 928.6710 & 0.0868 & 0.4323 \\
\hline Logistic II & 0.0023 & - & 0.3021 & -595.4801 & 1194.9602 & 0.7385 & 0.0000 \\
\hline Logistic III & 0.0000 & - & 0.0010 & -665.4880 & 1334.9760 & 0.7189 & 0.0000 \\
\hline Logistic IV & 0.9869 & 0.0000 & 0.0022 & -595.4802 & 1196.9604 & 0.5525 & 0.0000 \\
\hline $\begin{array}{l}\text { Gamma- } \\
\text { Logistic }\end{array}$ & 35.6785 & 1.9360 & 1.9360 & -456.3280 & 918.6560 & 0.0530 & 0.9388 \\
\hline
\end{tabular}




\section{ON THE GAMMA-LOGISTIC DISTRIBUTION}

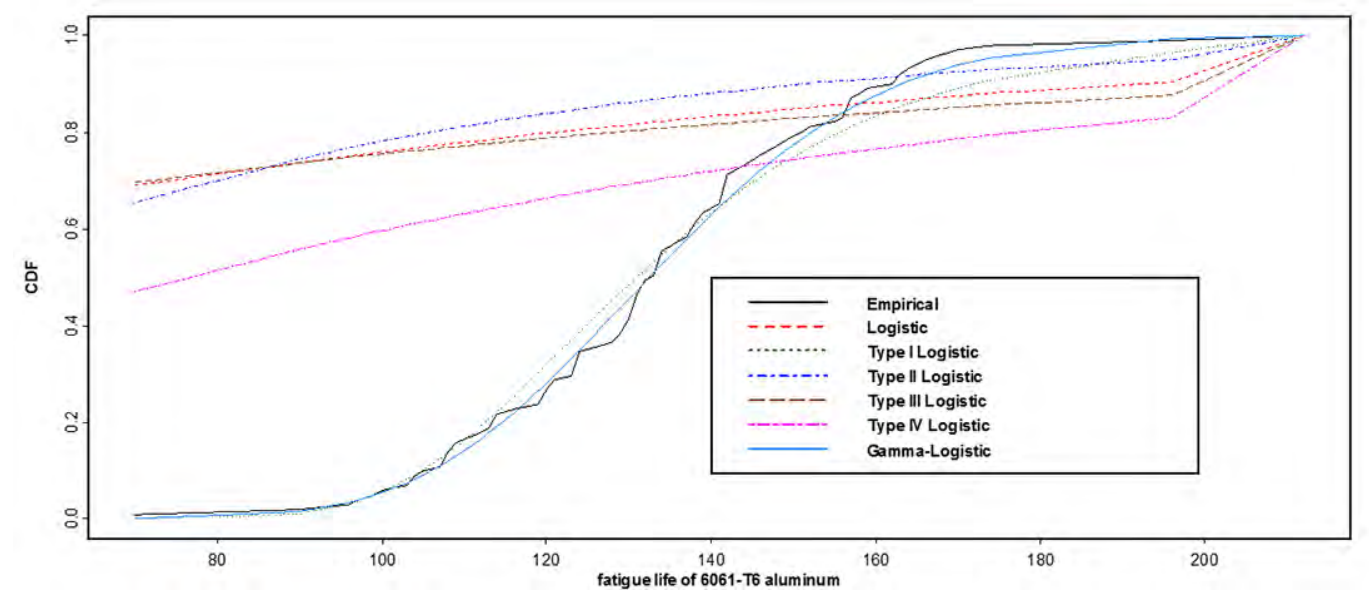

Figure 5. CDF for fitted distributions for fatigue life of 6061-T6 aluminum data

\section{Conclusion}

In this article, a special case of the gamma- $X$ family of distributions proposed by Alzaatreh, et al. (2013b), the gamma-logistic distribution is defined and studied. Various properties of the gamma-logistic distribution are studied, including moments, deviations from the mean and median, reliability parameter, Shannon entropy and order statistics. It is observed from figures 3 and 4 that the gammalogistic distribution exhibits a wide variety of skewness and kurtosis values and the distribution can be symmetric, right skewed or left skewed. A real data set is fitted to the gamma-logistic distribution and compared with other known distributions. The results show that the gamma-logistic distribution provides the best fit among the distributions.

\section{References}

Alzaatreh, A., Famoye, F. \& Lee, C. (2013a). Weibull-Pareto distribution and its applications. Communications in Statistics: Theory \& Methods, 42(9): 1673-1691. 


\section{ALZAATREH ET AL}

Alzaatreh, A., Famoye, F. \& Lee, C. (2012a). Gamma-Pareto distribution and its applications. Journal of Modern Applied Statistical Methods, 11(1): 78-94.

Alzaatreh, A. \& Ghosh, I. (2013). A study of the gamma-Pareto(IV) distribution and its applications. Revised and resubmitted to Communications in Statistics: Theory \& Methods.

Alzaatreh, A. \& Knight, K. (2013). On the gamma-half normal distribution and its applications. Journal of Modern Applied Statistical Methods, 12(1): 103119.

Alzaatreh, A., Lee, C. \& Famoye, F. (2013b). A new method for generating families of continuous distribution. Metron: International Journal of Statistics, 71(1): 63-79.

Alzaatreh, A., Lee, C. \& Famoye, F. (2012b). On the discrete analogues of continuous distributions. Statistical Methodology, 9(6): 589-603.

Arnold, B. C., Balakrishnan, N. \& Nagarajah, H. N. (2008): A first course in Order Statistics. New York: John Wiley.

Birirnaum, Z. W. and Saunders, S. C. (1969). A new family of life distributions. Journal of Applied Probability, 6: 319327.

Guess, F. \& Proschan, F. (1985). Mean residual life: theory and applications (No. FSU-STATISTICS-M702). Talahassee, FL: Florida State University Tallahassee Dept. of Statistics.

Hall, I. J. (1984). Approximate one-sided tolerance limits for the difference or sum of two independent normal variates. Journal of Qualitative Technology, 16: 15-19.

Hall, I. J., \& Wellner, J. A. (1979). Estimation of mean residual life. University of Rochester Department of Statistics Technical Report.

Johnson N. L., Kotz, S., and Balakrishnan N. (1994). Continuous Univariate Distributions, Volume 2. New York: John Wiley.

Kotz, S., \& Shanbhag, D. N. (1980). Some new approaches to probability distributions. Advances in Applied Probability, 12: 903-921.

Lee, C., Famoye, F. \& Alzaatreh, A. (2013). Methods for generating families of continuous distribution in the recent decades. Wiley Interdisciplinary Reviews: Computational Statistics, 5: 219-238.

Nadarajah, S., \& Pal, M. (2008). Explicit expressions for moments of gamma order statistics. Bulletin of the Brazilian Mathematical Society, New Series, 39: 45-60. 


\section{ON THE GAMMA-LOGISTIC DISTRIBUTION}

Shannon, E. C. (1948). A mathematical theory of communication. Bell System Technical Journal, 27: 379-432.

Weerahandi, S. \& Johnson, R. A. (1992). Testing reliability in a stress-

strength model when $\mathrm{X}$ and $\mathrm{Y}$ are normally distributed. Technometrics, 38: 83-91. 